\title{
3D Hierarchical Flower-Like Cobalt Ferrite Nanoclusters- Decorated Cotton Carbon Fiber anode with Improved Lithium Storage Performance
}

\author{
Yanshuang Meng ${ }^{1,3}$, Yulong Cheng ${ }^{1}$, Xinyou $\mathrm{Ke}^{2}$, Guofeng Ren ${ }^{2 *}$, and Fuliang $\mathrm{Zhu}^{1,3 *}$ \\ ${ }^{1}$ School of Materials Science and Engineering, Lanzhou University of Technology, Lanzhou 730050, China \\ ${ }^{2}$ Department of Mechanical and Aerospace Engineering, Case Western Reserve University, Clevleand, Ohio 44106, United States \\ ${ }^{3}$ State Key Laboratory of Advanced Processing and Recycling of Non-ferrous Metals, Lanzhou 730050, China
}

\begin{abstract}
The inverse spinel Cobalt ferrite $\left(\mathrm{CoFe}_{2} \mathrm{O}_{4}, \mathrm{CFO}\right)$ is considered to be a promising alternative to commercial graphite anodes for lithium ion batteries (LIBs). However, the further development of CFO is limited by its unstable structure during battery cycling and low electrical conductivity. In an effort to address the challenge, we construct three-dimensional hierarchical flower-like CFO nanoclusters (CFO NCs)-decorated carbonized cotton carbon fiber (CFO NCs/CCF) composite. This structure is consisted of microfibers and nanoflower cluster composited of CFO nanoparticle, in which CCF can be used as a long-range conductive matrix, while flower-like CFO NCs can provide abundant active sites, large electrode/electrolyte interface, short lithium ion diffusion path, and alleviated structural stress. As anode materials in LIBs, the flower-like CFO $\mathrm{NCs} / \mathrm{CCF}$ exhibits excellent electrochemical performance. After 100 cycles at a current density of $0.3 \mathrm{~A} \mathrm{~g} \mathrm{~g}^{-1}$, the CFO NCs/ CCF delivers a discharge/charge capacity of $1008 / 990 \mathrm{mAh} \mathrm{g}^{-1}$. Even at a high current density of $15 \mathrm{~A} \mathrm{~g}^{-1}$, it still maintains a charge/discharge capacity of 362/361 $\mathrm{mAh} \mathrm{g}^{-1}$.
\end{abstract}

Keywords : Cobalt Ferrite Nanoflowers, Biomass Derived Carbon, Anode, Lithium Ion Batteries

Received : 18 November 2020, Accepted : 26 January 2021

\section{Introduction}

In the past few decades, energy storage technology has been improved due to the demand of various forms of energy. Many innovative energy storage devices such as flow batteries [1-3], lithium ion batteries (LIBs) [4-6], and lithium-sulfur batteries [7] have been developed. Among them, LIBs have been widely utilized as power sources in practical fields due to their high operated voltage, long service life, high energy density and design flexibility, etc. [8]. However, the commercial LIBs are gradually unable to meet the requirement of practical applications. One of the important reasons is that the commercial anode material for LIBs (i.e. graphite) has lower

*E-mail address: chzfl@126.com, gxr136@case.edu

DOI: https://doi.org/10.33961/jecst.2020.01648

This is an open-access article distributed under the terms of the Creative Commons Attribution Non-Commercial License (http://creativecommons.org/licenses/by-nc/4.0) which permits unrestricted non-commercial use, distribution, and reproduction in any medium, provided the original work is properly cited. capacity (372 $\left.\mathrm{mAh} \mathrm{g}^{-1}\right)$. Therefore, carbonaceous materials, transition metal oxides (TMOs) [9], transition metal nitrides (TMNs) [10], transition metal disulfides (TMSs) [11] and transition metal phosphide (TMPs) [12] and many others have been explored to seek high capacity. In the last few years, ternary transition metal oxides (TTMOs) have attracted researchers' attention, due to their elevated reversible capacity, improved structure stability and enhanced electronic conductivity compared with TMOs [13]. In addition, TTMOs also show significant advantages with respect to economic and environmental aspects. Nevertheless, TTMOs still exhibit unsatisfying rate performance and rapid capacity degradation during cycling resulted from low conductivity and serious volume change during $\mathrm{Li}^{+} \mathrm{de} /$ intercalation.

To date, many studies have been focusing on nanosized active materials with rational designed morphologies, e.g. multi-shell hollow nanospheres [14], platelets [15], nanofibers [16], nano-octahedron [17], 
york-shell [18], nanoclusters [19], etc. and hybrid architectures, which are mainly composited with nanocarbons, i.e. carbon coated film [20], graphene [14], carbon nanotube [21], etc. These approaches can alleviate structural strain during $\mathrm{Li}^{+}$de/intercalation and enhance the charge transfer kinetic [22]. For examples, Qi et al. obtained controllable multi-shell cobalt ferrite nanospheres by controlling the calcination conditions of the precursor, which has a capacity retention rate of $88.9 \%$ (at $1 \mathrm{~A} \mathrm{~g}^{-1}$ ) and $86.9 \%$ (at $5 \mathrm{~A}$ $\left.\mathrm{g}^{-1}\right)$ after 500 cycles [14]. Zhang et al. synthesized a network of interconnected ferrite nanospheres with carbon nanotubes, which exhibited excellent cycling performance due to the stable ion/electron transfer pathway and alleviated volume change provided by the network structure [21]. Recently, three-dimensional (3D) micro-architectures have drawn many attentions, which typically are composed of lowdimensional building blocks (such as 2D nanosheets). 3D micro-architectures demonstrates diverse advantages, such as short $\mathrm{Li}^{+}$diffusion distance, large contact areas with electrolytes, as well as abundant active sites [23-25]. In addition, 3D microarchitectures can prevent self-aggregations of active materials, maintain the contact area with the electrolyte, as well as alleviate the volume stress induced by the electrochemical reaction [26]. Therefore, it is likely to boost the electrochemical performance of TTMOs by constructing a hierarchical hybrid structure composed of 3D micro-architecture assembled by low-dimensional nanostructured TTMOs on a carbon matrix. Biomass-derived carbon appears to be a desirable matrix to support nanostructured TTMOs [27], due to low cost and abundance of biomass, as well as the facile synthesis processes. Among biomass, cotton fibers are abundant, sustainable and green biomass carbon source in nature and could act as a matrix and provide a long-range electron transport pathway [28], eventually increasing the utilization of the active material.

Herein, we prepared a 3D hierarchical structure, carbonized cotton fibers decorated with flower-like $\mathrm{CoFe}_{2} \mathrm{O}_{4}$ nanoclusters (CFO NCs/CCF), by facilely mixing metal salt precursors and carbonized cotton fibers by stirring and subsequent calcination. In the $\mathrm{CFO} \mathrm{NCs} / \mathrm{CCF}$, the nanoflower-like CFO NCs anchored on the surface of carbon fibers are assembled by nano-petals composed of nanoparticles. The CFO NCs/CCF exhibits an excellent cycle and high rate performance. In term of cycling performance, the CFO NCs/CCF exhibits a specific discharge/charge capacity was $1008 / 990 \mathrm{mAh} \mathrm{g}^{-1}$ after the $100^{\text {th }}$ cycle at a current density of $0.3 \mathrm{~A} \mathrm{~g}^{-1}$. In term of rate performance, it displays a discharge/charge specific capacity of $362 / 361 \mathrm{mAh} \mathrm{g}^{-1}$ at a current density of $15 \mathrm{~A} \mathrm{~g}^{-1}$. The good electrochemical performance is ascribed to the synergistic effect of CCF and CFO $\mathrm{NCs}$ in the unique 3D hierarchical structure. On one hand, $\mathrm{CCF}$ can serve as a long-range conductive matrix to alleviate the inherent low conductivity of ferrite. On the other hand, CFO NCs can not only provide abundant active sites, short diffusion pathway for $\mathrm{Li}^{+}$, large contact interface between electrolyte and active materials, but also alleviate structural stress stemmed from electrochemical reactions.

\section{Experimental approaches}

\subsection{Material preparations}

All chemical reagents were analytical purity and not further purified.

\subsubsection{Preparation of cotton carbon fiber (CCF)}

$2 \mathrm{~g}$ of absorbent cotton fibers (Jiangxi Huazhong Textile Co., Ltd., China) was ultrasonically washed in deionized water and absolute ethanol for 1 hour, and then dried in a vacuum oven at $80^{\circ} \mathrm{C}$ for 12 hours. The washed absorbent cotton fibers were carbonized in an argon-containing vertical tube furnace at $800^{\circ} \mathrm{C}$ for 2 hours and the resulted product is designated as CCF.

2.1.2 Preparation of pure $\mathrm{CoFe}_{2} \mathrm{O}_{4}$ (p-CFO) and 3D hierarchical flower-like $\mathrm{CoFe}_{2} \mathrm{O}_{4}$ nanoclusters/ CCF (CFO NCs/CCF) composite

$100 \mathrm{mg}$ of CCF was dispersed into a methanol ((Aladdin Industrial Corporation, China) solution containing $0.5 \mathrm{mmol}$ of $\mathrm{Co}\left(\mathrm{NO}_{3}\right)_{2} \times 6 \mathrm{H}_{2} \mathrm{O}$ (Aladdin Industrial Corporation, China) and $1 \mathrm{mmol}$ of $\mathrm{Fe}\left(\mathrm{NO}_{3}\right)_{3} 9 \mathrm{H}_{2} \mathrm{O}$ (Aladdin Industrial Corporation, China) and stirred at $30^{\circ} \mathrm{C}$ for 12 hours. The remaining liquid was then evaporated in a vacuum oven at $30^{\circ} \mathrm{C}$ to obtain a precursor, which subsequently was calcined at $550^{\circ} \mathrm{C}$ for 2 hours in an argon-filled vertical tube furnace to obtain $\mathrm{CFO} \mathrm{NCs} / \mathrm{CCF}$. The synthesis of p-CFO was consistent with the above process except that no CCF powder was added. 


\subsection{Structural characterization}

The crystal structure of as-prepared material was obtained by the powder X-ray diffraction (XRD, D/ max-2400, Rigaku) with $\mathrm{Cu} \mathrm{K}_{\mathrm{a}}$ radiation $(1=$ $1.5418 \AA$ ) at $40 \mathrm{kV}$ and $100 \mathrm{~mA}$. Morphology observation was performed on scanning electron microscope (SEM, SIGMA HD, ZEISS) with an energy dispersive X-Ray spectrometer (EDS Inca X-Max, Oxford Instruments). Microstructure was analyzed by transmission electron microscope (TEM, JEM1200EX, JEOL). The Raman spectra of the sample were obtained on a microscopic confocal Raman spectrometer (LabRAM HR Evolution, HORIBA Jobin Yvon). The surface area and porosity of the samples were obtained from the nitrogen adsorption/ desorption isotherm using an automatic specific surface and porosity analyzer (MICROMERITICS ASAP 2460).

\subsection{Electrochemical characterizations}

Electrochemical performance measurements were carried out using CR2032 coin-type cells. The active material (CCF, p-CFO, or CFO NCs/CCF), the conductive agent (Acetylene black), and the binder (Polyvinylideneuf fluoride, PVDF) were placed in an agate mortar with a mass ratio of 7: 2: 1, and ground with a solvent of $\mathrm{N}$-methylpyrrolidone (NMP) to form a homogeneous slurry. The slurry was coated on a copper foil, and dried in a vacuum oven at $80^{\circ} \mathrm{C}$ for 12 hours. The coated copper foil was cut with a slicer to obtain working electrode discs (about $12 \mathrm{~mm}$ in diameter, with active material mass loading of $0.6 \mathrm{mg}$ ). The assembly of the half-cell was carried out in an argon-filled glove box $\left(\mathrm{H}_{2} \mathrm{O}\right.$ and $\mathrm{O}_{2}$ are less than $0.1 \mathrm{ppm}) .1 \mathrm{M} \mathrm{LiPF}_{6}$ in a mixture of ethylene carbonate (EC) and diethyl carbonate (DEC) (1:1 vol $\%$ ) was used as the electrolyte. Lithium foil was used as counter electrode, and Celgard 2400 microporous monolayer Polypropylene (PP) film with a thickness of $25 \mathrm{~mm}$ was used as the separator. Galvanostatic charge/discharge test was performed on a LAND CT2001A cycler. Cyclic voltammetry (CV) curves at various scan rates and electrochemical impedance spectra (EIS) in a frequency ranging from $100 \mathrm{kHz}$ to $0.01 \mathrm{~Hz}$ were recorded on an electrochemical workstation (CHI 660e, Chenhua, Shanghai).

\section{Results and Discussion}

\subsection{Materials synthesis and characterization}

Fig. 1 is the schematic diagram of the facile synthesis process of CFO NCs/CCF. First, the absorbent cotton fibers are carbonized into $\mathrm{CCF}$ by heat-treatment in Argon at $800^{\circ} \mathrm{C}$ for two hours. The high temperature ensures complete carbonization as well as high integrity of the cotton fibers, which ensures a high electrode integrity and fast electron pathways [29]. To prepare CFO NCs/CCF, CCF is dispersed in a solution of cobalt nitrate and ferric nitrate in methanol by stirring in a water bath at a temperature of $30^{\circ} \mathrm{C}$. The low surface tension of methanol, together with the capillary effect render enable CCF to be sat-

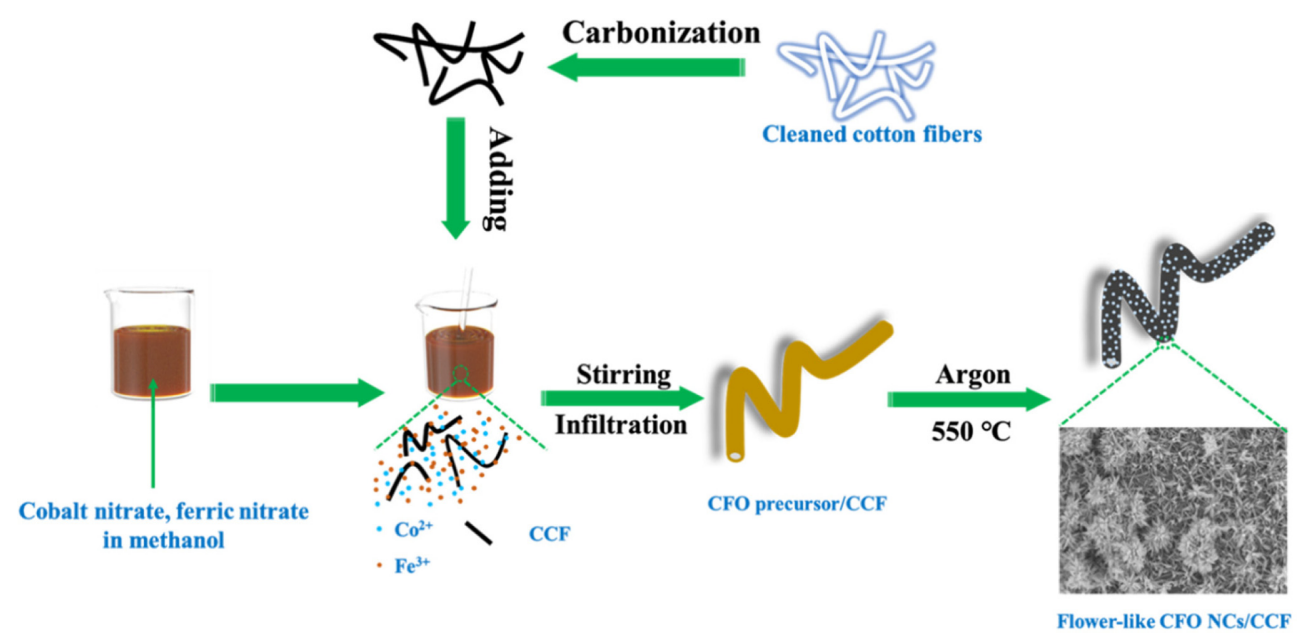

Fig. 1. Schematic diagram of the formation process of flower-like CFO NCs/CCF composite 
urated with the CFO precursor solution. After agitation for some time, the residual liquid was completely evaporated in an oven to obtain a CFO precursor/CCF composite. At last, the composite was calcined in argon to obtain $\mathrm{CFO} \mathrm{NCs} / \mathrm{CCF}$, during which, nitrates are decomposed and converted to CFO NCs.

Fig. S1 shows the XRD patterns of the CCF, p$\mathrm{CFO}$ and $\mathrm{CFO} \mathrm{NCs} / \mathrm{CCF}$. The broad peaks at $22^{\circ}$ in the diffraction patterns of $\mathrm{CCF}$ and $\mathrm{CFO} \mathrm{NCs} / \mathrm{CCF}$ are the characteristic peak of disordered carbon materials [30]. The peaks at $30.00^{\circ}, 35.34^{\circ}, 42.80,53.33^{\circ}$, $56.81^{\circ}, 62.47^{\circ}$, and $73.96^{\circ}$ in the diffraction patterns of the p-CFO and CFO NCs/CCF correspond to (220), (311), (400), (422), (511), (440), and (533) crystal planes of $\mathrm{CoFe}_{2} \mathrm{O}_{4}$ (PDF\# 22-1086, Fd-3m), respectively [31]. The above results indicate the successful synthesis of CFO on CCF. The Raman spectrum of CFO NCs/CCF was recorded and the result is shown in Fig. S2, and the inset is a partial amplification with a wavenumber of $150-750 \mathrm{~cm}^{-1}$. The weak peaks in $150-750 \mathrm{~cm}^{-1}$ correspond to the Raman activity modes of $\mathrm{CoFe}_{2} \mathrm{O}_{4}$, where the peaks of $308 \mathrm{~cm}^{-1}$ and $472 \mathrm{~cm}^{-1}$, namely $E_{\mathrm{g}}$ and $\mathrm{T}_{2 \mathrm{~g}}$, represents the motion of $\mathrm{O}$ ions, while the peak at $684 \mathrm{~cm}^{-1}$, namely $\mathrm{A}_{1 \mathrm{~g}}$, represents the octahedral and tetrahedral sites ions [32]. The $D, G$ and $D+G$ bands are characteristic bands of carbon materials. The D band at $1357 \mathrm{~cm}^{-1}$ corresponds to the disordered and defective regions of the $\mathrm{sp}^{3}$-bonded carbon and $\mathrm{G}$ band at $1601 \mathrm{~cm}^{-1} \mathrm{G}$ band corresponds to in-plane stretching of ordered $\mathrm{sp}^{2}$ bonded graphitic crystallites [23]. The Raman further confirms the successful synthesis of CFO NCs/ $\mathrm{CCF}$, in agreement with XRD patterns.

To investigate morphologies of synthesized samples, SEM observation was conducted and the images of $\mathrm{CCF}$ and $\mathrm{CFO} \mathrm{NCs} / \mathrm{CCF}$ are shown in Fig. 2. The SEM images of CCF show fibers with a diameter of approximately $10 \mu \mathrm{m}$ and a length of tens of micros (see Fig. 2a). Some fibers have a hollow structure (see Fig. 2b). Flower-like CFO NCs are successfully loaded on CCF, shown in Fig. 2c-e. The flower like CFO NCs are assembled from many nanorods with a diameter of tens of nanometers and a length of 100-

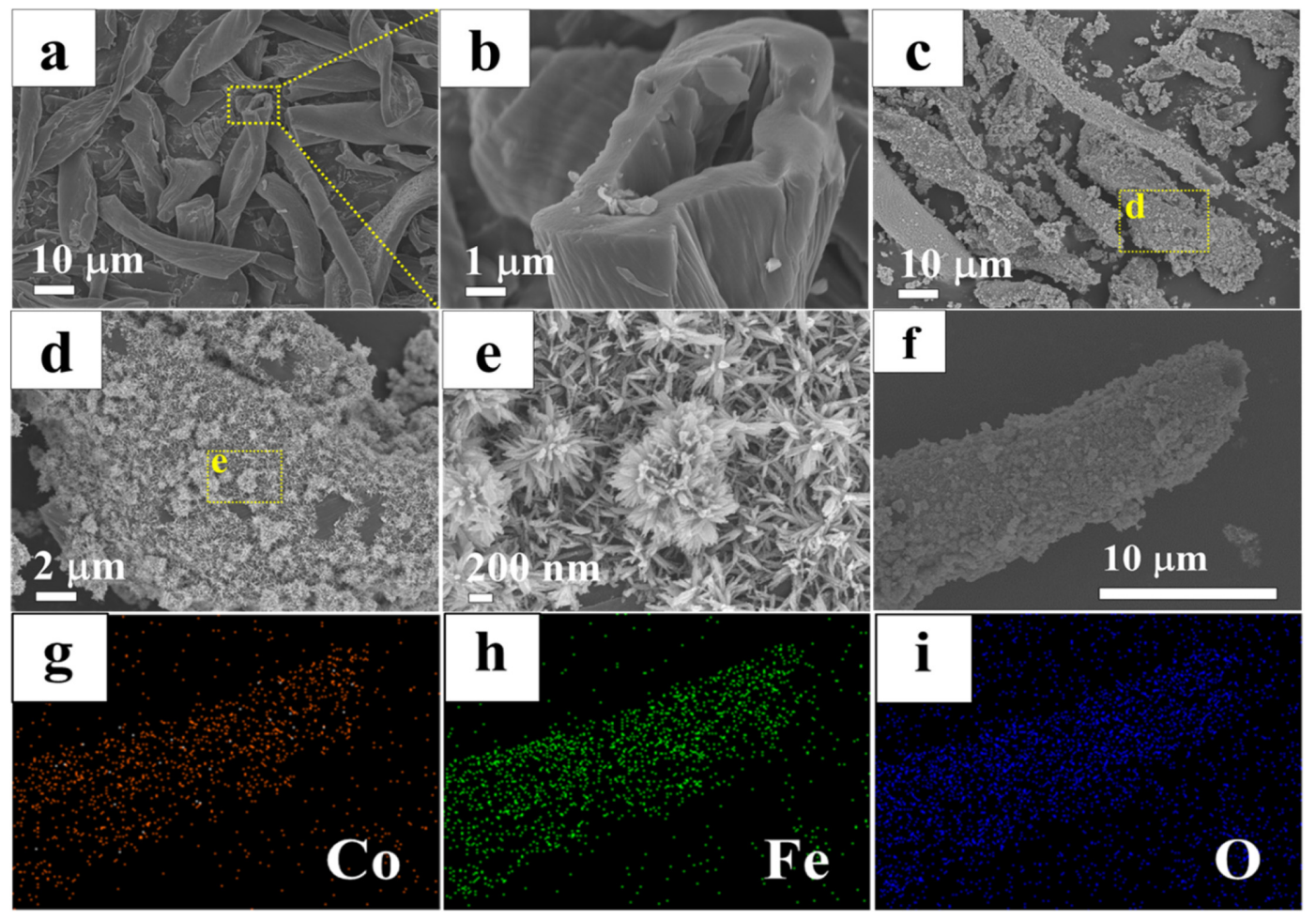

Fig. 2. SEM images of $\mathrm{CCF}(\mathrm{a}, \mathrm{b})$, flower-like $\mathrm{CFO} \mathrm{NCs} / \mathrm{CCF}$ composite (c-e) and Fe, Co. and $\mathrm{O}$ elements mapping of flower-like $\mathrm{CFO} \mathrm{NCs} / \mathrm{CCF}$ composite (f-i). 


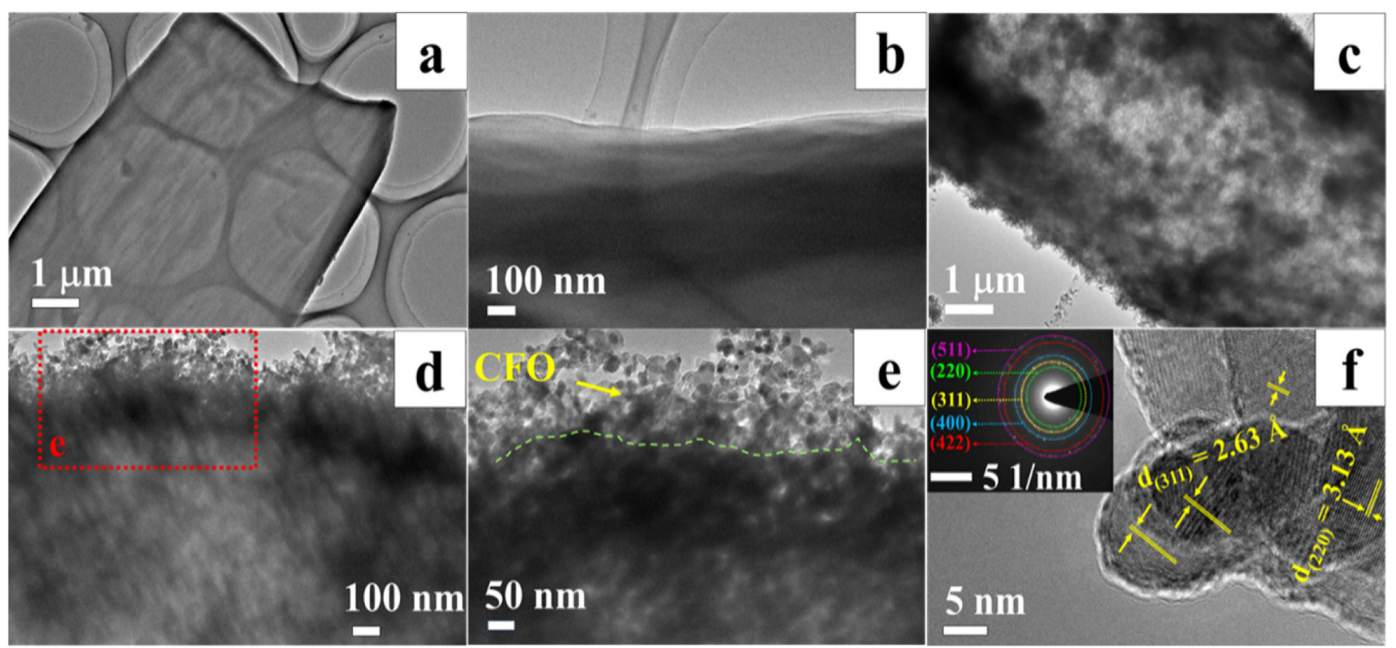

Fig. 3. TEM images of $\operatorname{CCF}(a, b)$ and flower-like $\mathrm{CFO} N \mathrm{NC} / \mathrm{CCF}$ composite (c-e), HRTEM image of flower-like CFO $\mathrm{NCs} / \mathrm{CCF}$ composite (f).

300 nanometers (see Fig. 2e). Element mappings of $\mathrm{Co}, \mathrm{Fe}$, and $\mathrm{O}$ as shown in Fig. $2 \mathrm{f}-\mathrm{i}$ indicate that flower-like CFO NCs grow uniformly on the surface of CCF. In this unique hierarchical architecture, CCF could provide long-range electron transport pathway, and CFO NCs can provide abundant active sites for $\mathrm{Li}^{+}$storage and large contact interface with electrolyte, as well as alleviate structural stress during $\mathrm{Li}^{+}$ intercalation.

TEM characterization was conducted to further observe the microstructure of the synthesized samples. The TEM images of CCF and CFO NCs/CCF are shown in Fig. 3, illustrating that the $\mathrm{CoFe}_{2} \mathrm{O}_{4}$ clusters were consisted of nanoparticles with diameter of $\sim 20 \mathrm{~nm}$ and successfully loaded on the CCF surface. The HRTEM image of the CFO NCs is shown in Fig. 3f, and the inset is the corresponding selected area electron diffraction (SAED) pattern. In HRTEM image, the lattice fringes with the interplanar spacing of $2.63 \AA$ and $3.13 \AA$ belong to the (311) and (220) crystal planes of CFO, respectively. The SAED pattern has five diffraction rings, corresponding to the (220), (311), (400), (422) and (511) planes of the crystalline CFO phase. The $\mathrm{N}_{2}$ adsorption/ desorption isotherm curves are shown in Fig. S3, in order to study the porosity of CFO NCs/CCF. The Brunauer-Emmett-Teller (BET) specific surface area of CFO NCs/CCF is calculated to be $181 \mathrm{~m}^{2} / \mathrm{g}$. And pore size is concentrated at $11 \mathrm{~nm}$. The large specific surface area and mesoporous nature of CFO NCs/ CCF could facilitate the penetration of the electrolyte.

\subsection{Electrochemical characterization}

To evaluate the electrochemical performance of the $\mathrm{CFO} \mathrm{NCs} / \mathrm{CCF}, \mathrm{CV}$ measurement was conducted and the results are shown in Fig. 4. According to previous reports [14, 33, 34], the lithiation/delithiation behavior of $\mathrm{CoFe}_{2} \mathrm{O}_{4}$ in LIBs can be described by the following equations:

$$
\begin{aligned}
& \mathrm{CoFe}_{2} \mathrm{O}_{4}+8 \mathrm{Li}^{+}+8 e^{-} \rightarrow \mathrm{Co}^{0}+2 \mathrm{Fe}^{0}+4 \mathrm{Li}_{2} \mathrm{O} \\
& \mathrm{Co}^{0}+2 \mathrm{Li}_{2} \mathrm{O} \leftrightarrow \mathrm{CoO}+2 \mathrm{Li}^{+}+2 e^{-} \\
& 2 \mathrm{Fe}^{0}+3 \mathrm{Li}_{2} \mathrm{O} \leftrightarrow \mathrm{Fe}_{2} \mathrm{O}_{3}+6 \mathrm{Li}^{+}+6 e^{-} \\
& \mathrm{CoO}+\mathrm{Fe}_{2} \mathrm{O}_{3}+8 \mathrm{Li}^{+}+8 e^{-} \rightarrow \mathrm{Co}^{0}+2 \mathrm{Fe}^{0}+4 \mathrm{Li}_{2} \mathrm{O}
\end{aligned}
$$

During the $1^{\text {st }}$ cathodic process, a sharp peak appears at $0.676 \mathrm{~V}$, corresponding to the conversion of $\mathrm{CoFe}_{2} \mathrm{O}_{4}$ to metallic $\mathrm{Co}^{0}$ and $\mathrm{Fe}^{0}$ (see Eq. 1) and formation of solid-electrolyte interphase (SEI) layer. In the subsequent $1^{\text {st }}$ anodic process, a broad peak appears around $1.601 \mathrm{~V}$, corresponding to the conversion of metallic $\mathrm{Co}^{0}$ and $\mathrm{Fe}^{0}$ to $\mathrm{CoO}$ and $\mathrm{Fe}_{2} \mathrm{O}_{3}$ (see Eqs. 2 and 3). During the cathodic process of the $2^{\text {nd }}$ and $3^{\text {rd }}$ cycles, two peaks with respect to the conver- 

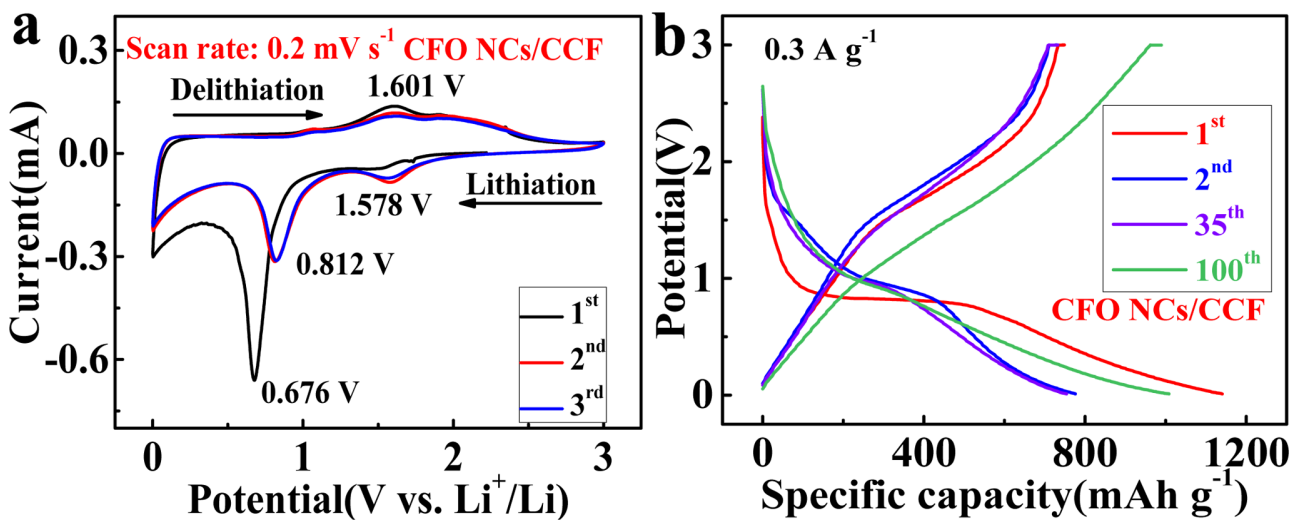

Fig. 4. CV curves in the first three cycles at a sweep rate of $0.2 \mathrm{mV} \mathrm{s}^{-1}$ (a), galvanostatic charge and discharge curves in the $1^{\text {st }}, 2^{\text {nd }}, 35^{\text {th }}$ and $100^{\text {th }}$ cycle at $0.3 \mathrm{~A} \mathrm{~g}^{-1}$ (b) of flower-like CFO NCs/CCF composite.

sion of $\mathrm{Fe}_{2} \mathrm{O}_{3}$ and $\mathrm{CoO}$ to metallic $\mathrm{Fe}^{0}$ and $\mathrm{Co}^{0}$ occurred at $0.812 \mathrm{~V}$ and $1.578 \mathrm{~V}$, respectively, while the positions of the peaks in anodic process is consistent with the $1^{\text {st }}$ cycle (see Equations 2 and 3). Notably, $\mathrm{CoFe}_{2} \mathrm{O}_{4}$ is re-arranged after the $1^{\text {st }}$ lithiation, $\mathrm{CoO}$ and $\mathrm{Fe}_{2} \mathrm{O}_{3}$ cannot be restored to the original spinel architecture, which is similar to other ferrites (e.g. zinc ferrite [35], nickel ferrite [16], manganese ferrite [36], etc.). Therefore, the lithiation/delithiation except for the first cycle was achieved by the mutual reversible conversion between $\mathrm{Co}^{0} / \mathrm{Fe}^{0} / \mathrm{Li}_{2} \mathrm{O}$ and $\mathrm{CoO} / \mathrm{Fe}_{2} \mathrm{O}_{3}$ (see Eq. 4). The $\mathrm{CV}$ curves of the $2^{\text {nd }}$ and $3^{\text {rd }}$ cycles tend to overlapping, suggesting an excellent reversibility of the electrochemical reaction.

Fig. $4 \mathrm{~b}$ shows discharge-charge curves of flowerlike CFO NCs/CCF composite for the $1^{\text {st }}, 2^{\text {nd }}, 35^{\text {th }}$, and $100^{\text {th }}$ cycle at $0.3 \mathrm{~A} \mathrm{~g}^{-1}$ with a potential window of 0.01 to $3 \mathrm{~V}$. The apparent loss of capacity in the first cycle is attributed to the formation of the SEI film and irreversible electrochemical conversion reaction $[17,37,38]$ Besides, the specific discharge/ charge capacity of the $2^{\text {nd }}, 35^{\text {th }}$ and $100^{\text {th }}$ cycle are 775.6/729.7, 754.1/732.4 and 1008.1/990.2 $\mathrm{mAh} \mathrm{g}^{-1}$, respectively.

Fig. 5a shows the cycling performance of the three samples at $0.3 \mathrm{~A} \mathrm{~g}^{-1}$. The slight decrease in the reversible specific capacity of the $\mathrm{CFO} \mathrm{NCs} / \mathrm{CCF}$ is assigned to the lithiation-induced mechanical attenuation and unstable SEI film in the first few cycles [17]. After the $100^{\text {th }}$ cycle, the reversible discharge/ charge specific capacity of the CFO NCs/CCF is maintained at $1008.2 / 990.1 \mathrm{mAh} \mathrm{g}^{-1}$. In contrast, the
CCF electrode exhibits stable but low capacity with cycling. The p-CFO electrode exhibits a rapid capacity decay with cycling. The increasing of capacity with cycling for nano-architectured transition metal oxide anodes has also been reported in previous studies [39-41]. Such an increase in capacity may be caused by following reasons: firstly, active material particles are gradually pulverized under electrochemical grinding effect, and forming a reversible growth/ decomposition polymeric gel-like film (PGF) on newly formed surfaces to provide additional lithium storage capacity alleged pseudo-capacitive behavior $[15,41,42]$. Furthermore, electrolyte penetrates into the porous structure of the solid electrode with cycling and lead to electrochemically activate more active materials $[8,43]$. In addition, CCF acts as a conductive and buffer matrix to improve the cycling stability of the electrode.

The rate performance of the three samples is shown in Fig. 5b. The CFO NCs/CCF has superior performance over CCF and p-CFO. Reversible discharge/charge capacities of 762.8/716.6 $\left(3^{\text {rd }}\right)$ and $703.7 / 682.6\left(9^{\text {th }}\right), 636 / 621.2\left(14^{\text {th }}\right), 470.5 / 464.9$ $\left(19^{\text {th }}\right), 408.6 / 404.8\left(24^{\text {th }}\right), 362.7 / 361.0\left(29^{\text {th }}\right), 777.6 /$ $763.2\left(70^{\text {th }}\right) \mathrm{mAh} \mathrm{g}^{-1}$ correspond to the current densities of $0.3,0.6,1,5,10,15,0.3 \mathrm{~A} \mathrm{~g}^{-1}$ respectively. It is interesting to note that when the current density is reduced from $15 \mathrm{~A} \mathrm{~g}^{-1}$ to $0.3 \mathrm{Ag}^{-1}$, the specific capacity does not completely return to the initial value, but the specific capacity increases with cycling. This may be due to the formation of PGF as described above and accompanied by the activation process, and this phe- 

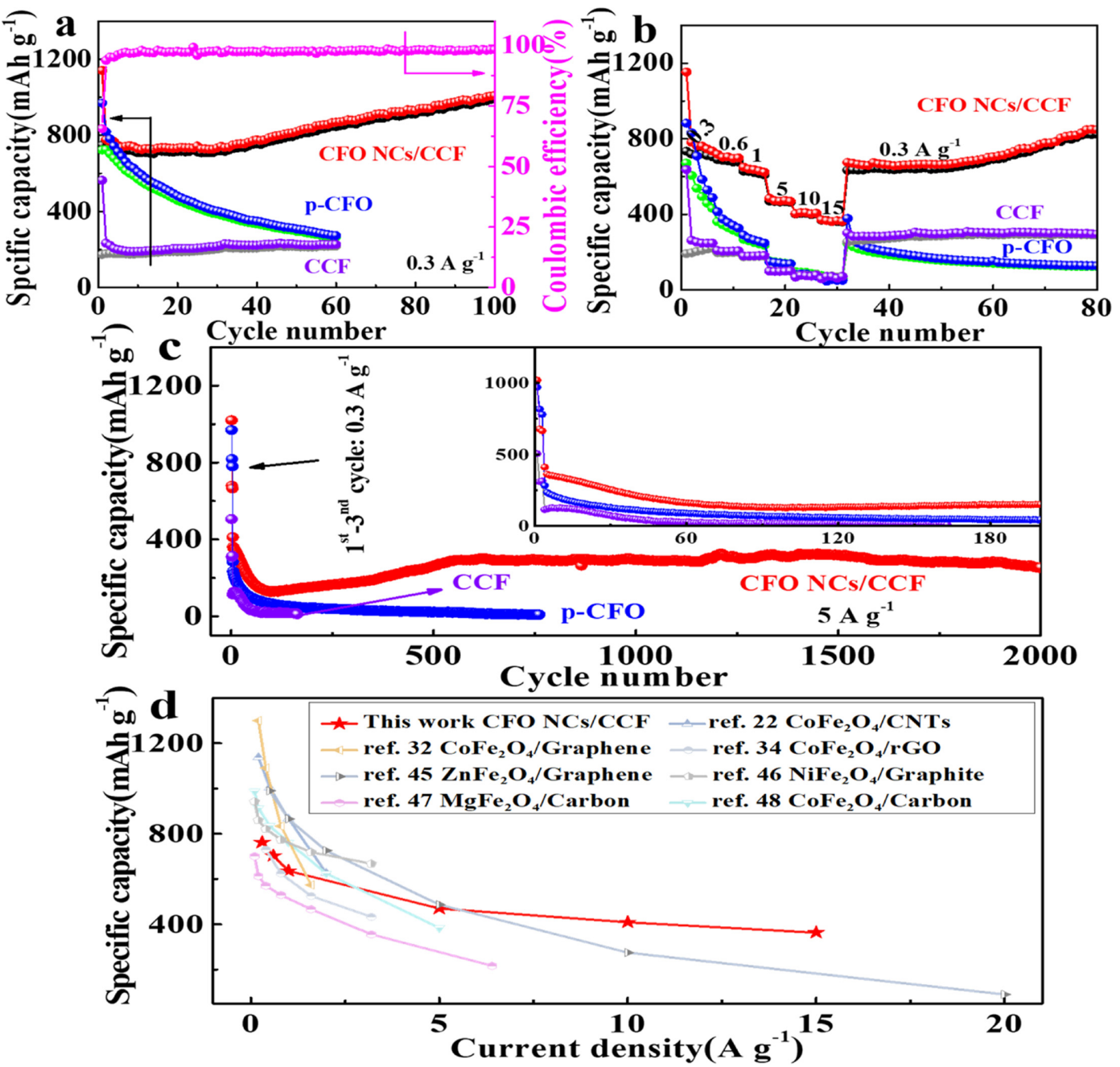

Fig. 5. Cyclic performance at $0.3 \mathrm{~A} \mathrm{~g}^{-1}$ (a), rate performance (b), Cyclic performance at $5 \mathrm{~A} \mathrm{~g}^{-1}$ (c) of CCF, p-CFO and $\mathrm{CFO} \mathrm{NCs} / \mathrm{CCF}$, and comparison of the rate performance of $\mathrm{CFO} \mathrm{NCs} / \mathrm{CCF}$ with previous cobalt or other ferrite-based anodes (d).

nomenon is similar to previous report [41].

To further test stability performance of $\mathrm{CCF}$, p$\mathrm{CFO}$ and $\mathrm{CFO} \mathrm{NCs} / \mathrm{CCF}$, long cycle performance at a current density of $5 \mathrm{~A} \mathrm{~g}^{-1}$ was carried out and the obtained data is shown in Fig. 5c (only for discharge specific capacity). In the first 80 cycles, the specific capacity of the CFO NCs/CCF fades severely. This might be caused by some CFO NCs that are detached from $\mathrm{CCF}$ or pulverized resulted from the high current density. The discharge specific capacity of CFO $\mathrm{NCs} / \mathrm{CCF}$ subsequently shows an increase until it reaches $290 \mathrm{mAh} \mathrm{g}^{-1}$ after 550 cycle, and maintains at $255.8 \mathrm{mAh} \mathrm{g}^{-1}$ after 2000 cycles. For $\mathrm{p}-\mathrm{CFO}$ and $\mathrm{CCF}$, their specific discharge capacities have been attenuated to $9.7\left(761^{\text {st }}\right)$ and $11.2\left(163^{\text {rd }}\right) \mathrm{mAh} \mathrm{g}^{-1}$ respectively. These results suggest that, the long-term cycling performance of $\mathrm{CFO} \mathrm{NCs} / \mathrm{CCF}$ is far superior to p-CFO and CCF. Fig. 5d shows the compassion of the rate performance of $\mathrm{CFO} \mathrm{NCs} / \mathrm{CCF}$ and other similar materials reported previously [21,31,33,44-47], suggesting that the $\mathrm{CFO} \mathrm{NCs} / \mathrm{CCF}$ has an excellent capacity retention and a satisfactory high rate performance. 

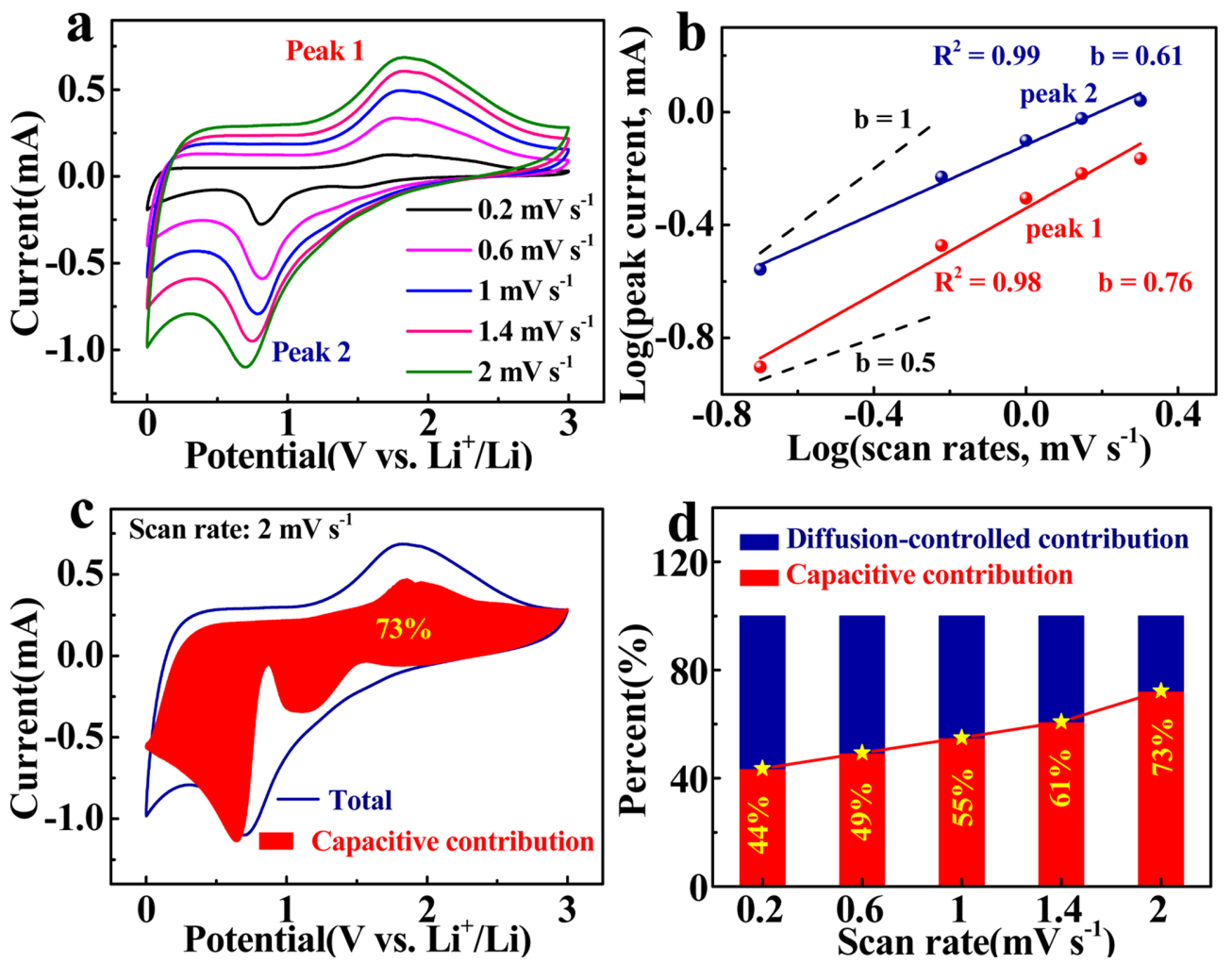

Fig. 6. $\mathrm{CV}$ curves of $\mathrm{CFO} \mathrm{NCs} / \mathrm{CCF}$ at various scan rates (a), the relationship between peak current and sweep rate (b), capacitive current of CFO NCs/CCF at a scan rate of $2 \mathrm{mV} \mathrm{s}^{-1}$ (c), and the contribution percentages of the capacitive and diffusion-controlled processes to charge storage of $\mathrm{CFO} \mathrm{NCs} / \mathrm{CCF}$ at various scan rates.

Some previous reports indicate that this unexceptional rate performance and cycle stability may be related to the capacitive behavior of nanomaterials with high surface area $[22,48]$. To further quantitatively examine the contribution of capacitive behavior to charge storage during the lithiation/de-lithiation process, $\mathrm{CV}$ of $\mathrm{CFO} \mathrm{NCs} / \mathrm{CCF}$ were performed at $0.2,0.6,1,1.4$, and $2 \mathrm{mV} \mathrm{s}^{-1}$, respectively (see Fig. $6 a)$. In general, the relationship between the peak current $(i)$ and the scan rate $(v)$ can be described as the following equations [49]:

$$
\begin{aligned}
& i=a v^{b} \\
& \log i=b \log v+\log a
\end{aligned}
$$

Where, $a$ and $b$ are adjustable parameters, $b$ ranges from 0.5 to 1 . When $b$ is close to 1 , it indicates that the capacitive behavior dominates. Capacity comes primarily from the process of diffusion-controlled when the $b$ is close to 0.5 . As shown in Fig. 6b, the values of $b$ at anodic and cathodic peaks are 0.76 and 0.61 , respectively, demonstrating that the capacity of CFO NCs/CCF is contributed from both the capacitive behavior and diffusion processes [49]. The total current in the CV scan is the sum of the capacitive current $\left(k_{1} v\right)$ and the diffusion-controlled current $\left(k_{1} v^{0.5}\right)$, described by the following equations [50]:

$$
\begin{aligned}
& i(V)=k_{1} v+k_{2} v^{0.5} \\
& (i(V)) /_{v^{0.5}}=k_{1} v^{0.5}+k_{2}
\end{aligned}
$$

The calculated results are shown in Fig. 6d. As the scan rate increases, the percentage of capacity from capacitive behavior increases. When the scan rate is 2 $\mathrm{mV} \mathrm{s}^{-1}$, the capacity contributed from capacitive behavior is $73 \%$, which is shown as the red area in 


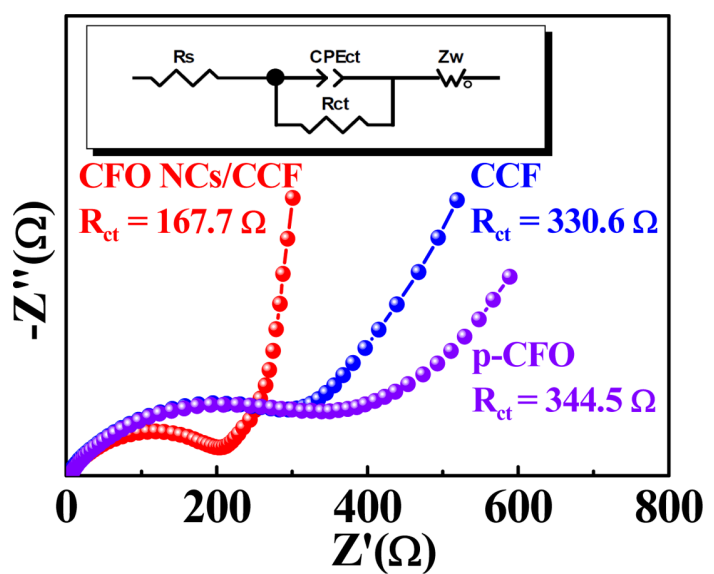

Fig. 7. Nyquist plots of p-CFO, CCF, and flower-like $\mathrm{CFO}$ $\mathrm{NCs} / \mathrm{CCF}$ composite before cycles (the inset is equivalent circuit model).

Fig. 6c (Others are in Fig. S4). The flower-like CFO $\mathrm{NCs}$ are composed of small nanoparticles of with high surface area, leading to a high capacitive induced capacity.

Electrochemical impedance spectroscopy (EIS) of $\mathrm{CCF}, \mathrm{p}-\mathrm{CFO}$ and $\mathrm{CFO} \mathrm{NCs} / \mathrm{CCF}$ was executed in the frequency range from $0.01 \mathrm{~Hz}$ to $100 \mathrm{kHz}$ (see Fig. 7). In Fig. 7, a typical Nyquist plot consists of a semicircle in the high frequency region and a straight line in the low frequency region. The insert illustration is the equivalent circuit model. Where $\mathrm{R}_{\mathrm{s}}$ is the intercept of the semicircle on the real axis representing the internal resistance of the battery, $\mathrm{R}_{\mathrm{ct}}$ is the semicircular diameter representing the charge transfer impedance with double layer capacitance $\mathrm{CPE}_{\mathrm{ct}}$, and $\mathrm{Z}_{\mathrm{w}}$ is the Warburg impedance of the ion diffusing into the interior of the bulk materials $[12,51]$. Clearly, the CFO NCs/CCF has a lower charge transfer impedance. The corresponding exchange current densities $\left(i^{0}\right)$ of CFO NCs/CCF, CCF, and p-CFO are $1.91 \times 10^{-2}$, $9.7 \times 10^{-3}, 9.31 \times 10^{-3} \mathrm{~mA} \mathrm{~cm}^{-2}$, calculated by the following equation:

$$
i^{0}=\frac{R T}{n F R_{c t}}
$$

In the above Eq. 9, $\mathrm{R}$ is the gas constant, $\mathrm{T}$ is the absolute temperature (take $298 \mathrm{~K}$ ), $\mathrm{n}$ is the number of electrons transferred in the redox reaction, $\mathrm{F}$ is the Faraday constant, and $\mathrm{R}_{\mathrm{ct}}$ is the charge transfer resistance. This result further confirms $\mathrm{CFO} \mathrm{NCs} / \mathrm{CCF}$ has improved electrode kinetics [8]. Compared to $\mathrm{CCF}$ and p-CFO, the higher slope of the CFO NCs/ $\mathrm{CCF}$ in the low frequency region also means a faster $\mathrm{Li}^{+}$diffusion rate [52]. In summary, explanations for the excellent electrochemical performance of 3D hierarchical flower-like CFO NCs/CCF composite might be as follows: (1) CCF acts as a long-range conductive matrix, providing an efficient electron transport channel and improving electrode reaction kinetics; (2) Flowerlike CFO NCs composed of nanoparticles of about 20 $\mathrm{nm}$ is able to reduce the diffusion path of $\mathrm{Li}^{+}$and alleviate volume stress during lithiation, and expand contact area with the electrolyte and (3) the high surface area of CFO NCs/CCF contribute the total capacity of the active materials by surface capacitive effect.

\section{Conclusions}

In this work, we have successfully grown flowerlike CFO NCs onto the surface of carbon fibers by a facile and scalable method. When CFO NCs/CCF is used as the anode of LIBs, it exhibits excellent rate performance and long cycle performance. $\mathrm{CFO} \mathrm{NCs/}$ $\mathrm{CCF}$ can deliver a capacity of $362.7 \mathrm{mAh} \mathrm{g}^{-1}$ at a current density of $15 \mathrm{~A} \mathrm{~g}^{-1}$ and maintain a capacity of $1008.2 \mathrm{mAh} \mathrm{g}^{-1}$ after 100 cycles at $0.3 \mathrm{Ag}^{-1}$. Such satisfactory electrochemical performance is primarily due to the synergistic effect of CFO NCs and CCF in this 3D hierarchical architecture. CCF can provide an effective electron transfer channel as a long-range conductive matrix, while flower-like CFO NCs can increase the electrolyte contact area, reduce the volume stress and ion diffusion path. This work provides a facile, low cost, and scalable approach for preparing this special architecture of 3D ferrite nanoflowers to decorate biomass-derived carbon materials as anode in lithium storage.

\section{Acknowledgement}

This work was supported by the National Natural Science Foundation of China (Grant No. 52064035), and the Natural Science Foundation of Gansu Province (Grant No. 20JR10RA166).

\section{Supporting Information}

Supporting Information is available at https:// doi.org/10.33961/jecst.2020.01648 


\section{Conflicts of interest}

There are no conflicts to declare.

\section{References}

[1] X. Ke, J. M. Prahl, J. I. D. Alexander, J. S. Wainright, T. A. Zawodzinski, R. F. Savinell, Chem. Soc. Rev., 2018, 47(23), 8721-8743.

[2] X. Ke, J. M. Prahl, J. I. D. Alexander, R. F. Savinell, J. Power Sources, 2018, 384, 295-302.

[3] X. Ke, J. I. D. Alexander, J. M. Prahl, R. F. Savinell, J. Power Sources, 2014, 270, 646-657.

[4] G. Ren, M. N. F. Hoque, J. Liu, J. Warzywoda, Z. Fan, Nano Energy, 2016, 21, 162-171.

[5] G. Ren, M. N. F. Hoque, X. Pan, J. Warzywoda, Z. Fan, J. Mater. Chem. A, 2015, 3(20), 10787-10794.

[6] Y. Li, Y. Meng, X. Liu, M. Xiao, Q. Hu, R. Li, X. Ke, G. Ren, F. Zhu, J. Power Sources, 2019, 442, 227256.

[7] G. Ren, S. Li, Z.-X. Fan, J. Warzywoda, Z. Fan, J. Mater. Chem. A, 2016, 4(42), 16507-16515.

[8] K. Cao, L. Jiao, H. Liu, Y. Liu, Y. Wang, Z. Guo, H. Yuan, Adv. Energy Mater, 2015, 5(4), 1401421.

[9] L. Yin, Y. J. Gao, I. Jeon, H. Yang, J.-P. Kim, S. Y. Jeong, C. R. Cho, Chem. Eng. J., 2019, 356, 60-68.

[10] S. Dong, X. Chen, X. Zhang, G. Cui, Coordin. Chem. Rev., 2013, 257(13-14), 1946-1956.

[11] M. Chhowalla, H. S. Shin, G. Eda, L. J. Li, K. P. Loh, H. Zhang, Nat. Chem., 2013, 5(4), 263-275.

[12] P. Zhu, Z. Zhang, P. Zhao, B. Zhang, X. Cao, J. Yu, J. Cai, Y. Huang, Z. Yang, Carbon, 2019, 142, 269-277.

[13] Y. Zhao, X. Li, B. Yan, D. Xiong, D. Li, S. Lawes, X. Sun, Adv. Energy Mater, 2016, 6(8), 1502175.

[14] Y. Qi, B. Liu, L. Zhang, Y. Huo, L. Li, H. Xie, C. Wang, Z. Su, J. Mater. Chem. A, 2017, 5(41), 21994-22003.

[15] Z. Zhang, W. Li, R. Zou, W. Kang, Y. San Chui, M. F. Yuen, C.-S. Lee, W. Zhang, J. Mater. Chem. A, 2015, 3(13), 6990-6997.

[16] C. T. Cherian, J. Sundaramurthy, M. V. Reddy, P. Suresh Kumar, K. Mani, D. Pliszka, C. H. Sow, S. Ramakrishna, B. V. R. Chowdari, ACS Appl. Mater. Inter, 2013, 5(20), 9957-9963.

[17] Y. Ma, Y. Ma, D. Geiger, U. Kaiser, H. Zhang, G.-T. Kim, T. Diemant, R. J. Behm, A. Varzi, S. Passerini, Nano Energy, 2017, 42, 341-352.

[18] L. Hou, R. Bao, Y. Zhang, X. Sun, J. Zhang, H. Dou, X. Zhang, C. Yuan, J. Mater. Chem. A, 2018, 6(37), 1794717958.

[19] X. Yao, J. Kong, C. Zhao, D. Zhou, R. Zhou, X. Lu, Electrochim. Acta, 2014, 146, 464-471.

[20] D. Bresser, E. Paillard, R. Kloepsch, S. Krueger, M. Fiedler, R. Schmitz, D. Baither, M. Winter, S. Passerini, Adv. Energy Mater, 2013, 3(4), 513-523.

[21] Z. Zhang, Y. Wang, M. Zhang, Q. Tan, X. Lv, Z. Zhong, F. Su, J. Mater. Chem. A, 2013, 1(25), 7444-7450.

[22] L. Zhang, T. Wei, Z. Jiang, C. Liu, H. Jiang, J. Chang,
L. Sheng, Q. Zhou, L. Yuan, Z. Fan, Nano Energy, 2018, 48, 238-247.

[23] L. Wan, D. Yan, X. Xu, J. Li, T. Lu, Y. Gao, Y. Yao, L. Pan, J. Mater. Chem. A, 2018, 6(48), 24940-24948.

[24] X. Li, X. Tian, T. Yang, Y. Song, Y. Liu, Q. Guo, Z. Liu, J. Alloy. Compd., 2018, 735, 2446-2452.

[25] M. Zhang, M. Jia, J. Alloy. Compd., 2013, 551, 53-60.

[26] W. Cao, W. Wang, H. Shi, J. Wang, M. Cao, Y. Liang, M. Zhu, Nano Res., 2018, 11(3), 1437-1446.

[27] M. Liu, H. Jin, E. Uchaker, Z. Xie, Y. Wang, G. Cao, S. Hou, J. Li, Nanotechnology, 2017, 28(15), 155603.

[28] Ruopian Fang , S. Zhao, Pengxiang Hou , Min Cheng, Shaogang Wang, Hui-Ming Cheng, Chang Liu , F. Li, Adv. Mater, 2016, 28(17), 3373-3382.

[29] S. H. Chung, C. H. Chang, A. Manthiram, Acs Nano, 2016, 10(11), 10462-10470.

[30] T. Chen, L. Pan, T. Lu, C. Fu, D. H. C. Chua, Z. Sun, J. Mater. Chem. A, 2014, 2(5), 1263-1267.

[31] S. Li, B. Wang, J. Liu, M. Yu, Electrochim. Acta, 2014, 129, 33-39.

[32] S. Shankar, M. Kumar, A. K. Ghosh, O. P. Thakur, M. Jayasimhadri, J. Alloy. Compd., 2019, 779, 918-925.

[33] K. Wu, D. Liu, Y. Tang, Electrochim. Acta, 2018, 263, 515-523.

[34] Z. Wang, P. Fei, H. Xiong, C. Qin, W. Zhao, X. Liu, Electrochim. Acta, 2017, 252, 295-305.

[35] Z. Xing, Z. Ju, J. Yang, H. Xu, Y. Qian, Nano Res., 2012, 5(7), 477-485.

[36] C. Zhang, C. Jin, G. Teng, Y. Gu, W. Ma, Chem. Eng. J., 2019, 365, 121-131.

[37] S. J. Yang, S. Nam, T. Kim, J. H. Im, H. Jung, J. H. Kang, S. Wi, B. Park, C. R. Park, J. Am. Chem. Soc., 2013, 135(20), 7394-7397.

[38] L. Qie, W. M. Chen, Z. H. Wang, Q. G. Shao, X. Li, L. X. Yuan, X. L. Hu, W. X. Zhang, Y. H. Huang, $A d v$. Mater, 2012, 24(15), 2047-2050.

[39] S. Zhu, J. Li, X. Deng, C. He, E. Liu, F. He, C. Shi, N. Zhao, Adv. Funct. Mater, 2017, 27(9), 1605017.

[40] F. Zou, X. Hu, Z. Li, L. Qie, C. Hu, R. Zeng, Y. Jiang, Y. Huang, Adv. Mater, 2014, 26(38), 6622-6628.

[41] D. Li, X. Li, S. Wang, Y. Zheng, L. Qiao, D. He, $A C S$ Appl. Mater. Inter. 2014, 6(1), 648-654.

[42] Z. Wang, D. Luan, S. Madhavi, Y. Hu, X. W. Lou, Energy Environ. Sci., 2012, 5(1), 5252-5256.

[43] X. Xu, H. Tan, K. Xi, S. Ding, D. Yu, S. Cheng, G. Yang, X. Peng, A. Fakeeh, R. V. Kumar, Carbon, 2015, $84,491-499$

[44] X. Yao, J. Kong, D. Zhou, C. Zhao, R. Zhou, X. Lu, Carbon, 2014, 79, 493-499.

[45] L. Qu, X. Hou, X. Huang, Q. Liang, Q. Ru, B. Wu, K.H. Lam, ChemElectroChem, 2017, 4(12), 3148-3155.

[46] C. Gong, Y.-J. Bai, Y.-X. Qi, N. Lun, J. Feng, Electrochim. Acta, 2013, 90, 119-127.

[47] L. Lu, X. Jiao, J. Fan, W. Lei, Y. Ouyang, X. Xia, Z. Xue, Q. Hao, Electrochim. Acta, 2019, 295, 461-471.

[48] D. Cao, Z. Yao, J. Liu, J. Zhang, C. Li, Energy Storage 
Mater, 2018, 11, 152-160.

[49] F. Liu, X. Cheng, R. Xu, Y. Wu, Y. Jiang, Y. Yu, $A d v$. Funct. Mater, 2018, 28(18), 1800394.

[50] J. Wang., J. Polleux., J. Lim., B. Dunn., J. Mater. Chem.
C, 2007, 111, 14925-14931.

[51] J. Wang, C. Wang, M. Zhen, Chem. Eng. J., 2019, 356, $1-10$

[52] C. Liao, S. Wu, Chem. Eng. J., 2019, 355, 805-814. 